\title{
Green Function for Gradient Perturbation of Unimodal Lévy Processes in the Real Line
}

\author{
T. Grzywny ${ }^{1}\left(\mathbb{C} \cdot\right.$ T. Jakubowski $^{1}\left[\right.$ ] $\cdot$ G. Żurek ${ }^{1}$ (])
}

Received: 5 February 2018 / Revised: 26 January 2019 / Published online: 18 February 2019

(c) The Author(s) 2019

\begin{abstract}
We prove that the Green function of the generator of symmetric unimodal Lévy process with the weak lower scaling order bigger than one and the Green functions of its gradient perturbations are comparable for bounded $C^{1,1}$ subsets of the real line if the drift function is from an appropriate Kato class.
\end{abstract}

Keywords Unimodal Lévy process · Poisson kernel $\cdot$ Green function $\cdot C^{1,1}$ domain

Mathematics Subject Classification Primary 47A55 - 60J75; Secondary 47G20 . $60 \mathrm{~J} 35 \cdot 60 \mathrm{~J} 50$

\section{Introduction}

Perturbations of Markovian generators are widely studied for many years. This theory may be considered from various points of view. Such perturbations appear, e.g., in local and non-local partial differential equations [10,11,33,34], semigroup theory [4, $7,8,26,30]$, stochastic processes [25,31,31] and potential theory $[9,12,17]$. One of the natural questions is how this perturbation affects the solutions of the equations related to the unperturbed operator (e.g., the transition density of the semigroup and the Green function).

Communicated by Fuad Kittaneh.

The first author was supported by the statutory fund of the Department of Mathematics, Faculty of Pure and Applied Mathematics, Wrocław University of Science and Technology.

The second and third authors were partially supported by NCN Grant 2015/18/E/ST1/00239.

$\bowtie \quad$ G. Żurek

grzegorz.zurek@pwr.edu.pl

1 Faculty of Pure and Applied Mathematics, Wrocław University of Science and Technology, Wrocław, Poland 
In this paper we are interested in the gradient perturbations and the potential theory of the perturbed operator. We briefly recall some results closely related to our research. Cranston and Zhao in [16] considered the operator $\Delta+b(x) \nabla$ in $\mathbb{R}^{d}$ for $d \geqslant 2$. They proved that the Green function and harmonic measures of Lipschitz domains are comparable with those of $\Delta$ for the drift $b$ from an appropriate Kato class. In [24] and [25] Jakubowski studied the $\alpha$-stable Ornstein-Uhlenbeck process. He proved estimates for the first exit time from the ball and Harnack inequality for this process. In [9] Bogdan and Jakubowski proved similar results as Cranson and Zhao for $\Delta^{\alpha / 2}+$ $b(x) \nabla$ in $C^{1,1}$ domains in $\mathbb{R}^{d}, d \geqslant 2$. In the recent paper [19] these results were generalized to the case of pure-jump symmetric unimodal Lévy processes possessing certain weak scaling properties. We note that in the papers $[9,19]$ the case $d=1$ was omitted. The aim of this paper is to fill this gap and prove analogous results in one-dimensional case.

We will denote by $\left\{X_{t}\right\}$ a pure-jump symmetric unimodal Lévy process on $\mathbb{R}$, that is, a process with the symmetric density function $p_{t}(x)$ on $\mathbb{R} \backslash\{0\}$ which is non-increasing on $\mathbb{R}_{+}$. The characteristic exponent of $\left\{X_{t}\right\}$ equals

$$
\psi(x)=\int_{\mathbb{R}}(1-\cos (x z)) \nu(\mathrm{d} z), \quad x \in \mathbb{R} .
$$

where $v$ is a Lévy measure, i.e., $\int_{\mathbb{R}}\left(1 \wedge|z|^{2}\right) v(\mathrm{~d} z)<\infty$. For general information on unimodal processes, we refer the reader to $[5,18,35]$. A primary example of the mentioned class of processes is the symmetric $\alpha$-stable Lévy process having the fractional Laplacian $\Delta^{\alpha / 2}$ as a generator.

Let

$$
\mathcal{L} f(x)=\int_{\mathbb{R}}\left(f(x+z)-f(x)-\mathbf{1}_{|z|<1}\left(z f^{\prime}(x)\right)\right) v(\mathrm{~d} z), \quad f \in C_{b}^{2}(\mathbb{R}),
$$

be the generator of the process $X_{t}$. We will consider a non-empty bounded open $C^{1,1}$ set $D$ and the Green function $G_{D}(x, y)$ for $\mathcal{L}$. Now, let $\tilde{G}_{D}(x, y)$ be the Green function for

$$
\tilde{\mathcal{L}}=\mathcal{L}+b(x) \frac{\mathrm{d}}{\mathrm{d} x}
$$

where $b$ is a function from the Kato class $\mathcal{K}_{1}$. (For definitions of $C^{1,1}$ sets, the Green function and the Kato class, we refer the reader to Sects. 2 and 4). Our main result is

Theorem 1.1 Let $b \in \mathcal{K}_{1}$, and let $D \subset \mathbb{R}$ be an union of finitely many open, bounded intervals with positive distance between every two intervals. We assume that the characteristic exponent

$$
\psi \in \operatorname{WLSC}(\underline{\alpha}, 0, \underline{c}) \cap \operatorname{WLSC}\left(\underline{\alpha}_{1}, 1, \underline{c}_{1}\right) \cap \operatorname{WUSC}(\bar{\alpha}, 0, \bar{C}), \quad \text { where } \underline{\alpha}_{1}>1 \text {. }
$$

Then, there exists a constant $C$ such that for $x, y \in D$,

$$
C^{-1} G_{D}(x, y) \leqslant \tilde{G}_{D}(x, y) \leqslant C G_{D}(x, y) .
$$


Here WLSC and WUSC are the classes of functions satisfying a weak lower and weak upper scaling condition, respectively (see Sect. 2 for definitions). Generally, we follow the approach of [9] and [19]; however, there are some important differences. Although the geometry of the set $D$ is much simpler than in higher dimensions, it seems that the one-dimensional case sometimes demands more delicate arguments. One of the main difficulties is to obtain the proper estimates for derivative of the Green function. We use them to show the uniform integrability of $\partial_{x} G_{D}(x, y)$ against $|b(z)| \mathrm{d} z$ (see Lemma 3.11). As it was mentioned in [9], for $d=1$, the available estimates

$$
\left|\partial_{x} G_{D}(x, y)\right| \leqslant c G_{D}(x, y) /\left(\delta_{D}(x) \wedge|x-y|\right)
$$

are not integrable near $y$. Estimates (1.3) hold, i.e., if $v^{\prime}(r) / r$ is non-increasing (see [19, Lemma 3.2] and [29, Theorem 1.4]). To overcome this difficulty, we improve estimates (1.3) near the pole in $y$, see Proposition 3.5 and Corollary 3.6. The obtained estimates of $\left|\partial_{x} G_{D}(x, y)\right|$ are summarized in Theorem 3.10. This result is new even for the fractional Laplacian. We emphasize here that we make no additional assumption on the monotonicity of $v^{\prime}(r) / r$ as mentioned above. Like in the mentioned papers, our main tool is the perturbation formula. First, we use it to obtain estimates for sets $D$ with a small radius. Since the Green function $G_{D}(x, y)$ is bounded, we do not use the perturbation series as in [9] and [19]. Instead, we propose a simpler iteration argument.

We note also that one of our standing assumptions is $\underline{\alpha}_{1}>1$. It may be understood that the rank of the operator $\mathcal{L}$ is larger than 1 . Without this assumption the drift term may have a stronger effect than $\mathcal{L}$ on the behavior of the Green function of the $\tilde{\mathcal{L}}$. Any results concerning the cases $\underline{\alpha} \leqslant 1$ would be interesting; however, for $\underline{\alpha}<1$, Theorem 1.1 cannot hold in the form above (see the Introduction of [9] for more details)

This paper is organized as follows. In Sect. 2 we define the process $X$ and present its basic properties. In Sect. 3, we introduce the Green function of $X$ and prove the estimates for its derivative and some 3G-like inequalities. In Sect. 4, we define the operator $\tilde{\mathcal{L}}$ and the Green function of the underlying Markov process. Lastly, in Sect. 5, we prove Theorem 1.1.

When we write $f(x) \stackrel{C}{\approx} g(x)$, we mean that there is a number $0<C<\infty$ independent of $x$, i.e., a constant, such that for every $x, C^{-1} f(x) \leqslant g(x) \leqslant C f(x)$. If the value of $C$ is not important we simply write $f(x) \approx g(x)$. The notation $C=$ $C(a, b, \ldots, c)$ means that $C$ is a constant which depends only on $a, b, \ldots, c$.

We use the convention that numbered constants denoted by capital letters do not change throughout the paper. For a symmetric function $f: \mathbb{R} \rightarrow[0, \infty)$ we shall often write $f(r)=f(x)$ for any $x \in \mathbb{R}$ with $|x|=r$.

\section{Preliminaries}

In what follows, $\mathbb{R}$ denotes the Euclidean space of real numbers and $d y$ stands for the Lebesgue measure on $\mathbb{R}$. Without further mention we will only consider Borel sets, measures and functions in $\mathbb{R}$. As usual, we write $a \wedge b=\min (a, b)$ and $a \vee b=$ 
$\max (a, b)$. We let $B(x, r)=\{y \in \mathbb{R}:|x-y|<r\}$. For the arbitrary set $A \subset \mathbb{R}$, the distance to the boundary of $A$ will be denoted by

$$
\delta_{x}^{A}=\operatorname{dist}(x, \partial A)
$$

To simplify the notation, while referring to the set $D$, we will omit the superscript, i.e.,

$$
\delta_{x}=\delta_{x}^{D}=\operatorname{dist}(x, \partial D) .
$$

Definition 1 Let $\underline{\theta} \in[0, \infty)$ and $\phi$ be a nonnegative nonzero function on $(0, \infty)$. We say that $\phi$ satisfies the weak lower scaling condition (at infinity) if there are numbers $\underline{\alpha}>0$ and $\underline{c} \in(0,1]$ such that

$$
\phi(\lambda \theta) \geqslant \underline{c} \lambda \underline{\underline{\alpha}} \phi(\theta) \text { for } \lambda \geqslant 1, \quad \theta>\underline{\theta} .
$$

In short, we say that $\phi$ satisfies $\operatorname{WLSC}(\underline{\alpha}, \underline{\theta}, \underline{c})$ and write $\phi \in \operatorname{WLSC}(\underline{\alpha}, \underline{\theta}, \underline{c})$. If $\phi \in \mathrm{WLSC}(\underline{\alpha}, 0, \underline{c})$, then we say that $\phi$ satisfies the global weak lower scaling condition.

Similarly, we consider $\bar{\theta} \in[0, \infty)$. The weak upper scaling condition holds if there are numbers $\bar{\alpha}<2$ and $\bar{C} \in[1, \infty)$ such that

$$
\phi(\lambda \theta) \leqslant \bar{C} \lambda^{\bar{\alpha}} \phi(\theta) \text { for } \lambda \geqslant 1, \quad \theta>\bar{\theta} .
$$

In short, $\phi \in \mathrm{WUSC}(\bar{\alpha}, \bar{\theta}, \bar{C})$. For global weak upper scaling we require $\bar{\theta}=0$ in (2.2).

Throughout the paper, $X_{t}$ will be a pure-jump symmetric unimodal Lévy process on $\mathbb{R}$. The Lévy measure $v$ of $X_{t}$ is symmetric and non-increasing, so it admits a density $v$, i.e., $v(d x)=v(|x|) d x$. Hence, the characteristic exponent $\psi$ of $X_{t}$ is symmetric as well.

We assume that (see Theorem 1.1)

$$
\begin{aligned}
& \psi \in \operatorname{WLSC}(\underline{\alpha}, 0, \underline{c}) \cap \operatorname{WUSC}(\bar{\alpha}, 0, \bar{C}), \\
& \psi \in \operatorname{WLSC}\left(\underline{\alpha}_{1}, 1, \underline{c}_{1}\right), \quad \text { for some } \underline{\alpha}_{1}>1 .
\end{aligned}
$$

Following [32], we define

$$
h(r)=\int_{\mathbb{R}}\left(1 \wedge \frac{|x|^{2}}{r^{2}}\right) v(|x|) \mathrm{d} x, \quad r>0 .
$$

Let us notice that

$$
h(\lambda r) \leqslant h(r) \leqslant \lambda^{2} h(\lambda r), \quad \lambda>1 .
$$


Moreover, by [5, Lemma 1 and (6)],

$$
2^{-1} \psi(1 / r) \leqslant h(r) \leqslant C_{1} \psi(1 / r)
$$

Here, we may choose $C_{1}=\pi^{2} / 2$, but it will be more convenient to write this constant as $C_{1}$. We define the function $V$ as follows,

$$
V(0)=0 \text { and } V(r)=1 / \sqrt{h(r)}, \quad r>0 .
$$

Since $h(r)$ is non-increasing, $V$ is non-decreasing. We have

$$
V(r) \leqslant V(\lambda r) \leqslant \lambda V(r), \quad r \geqslant 0, \lambda>1 \text {. }
$$

By weak scaling properties of $\psi$ and the property $h(r) \approx \psi(1 / r)$, we get

$$
\begin{gathered}
\left(\frac{\underline{c}}{2 C_{1}}\right)^{1 / 2} \lambda^{\underline{\alpha} / 2} \leqslant \frac{V(\lambda r)}{V(r)} \leqslant\left(2 \bar{C} C_{1}\right)^{1 / 2} \lambda^{\bar{\alpha} / 2}, \quad r>0, \lambda>1, \\
\frac{V(\eta r)}{V(r)} \leqslant\left(\frac{2 C_{1}}{\underline{c}_{1}}\right)^{1 / 2} \eta^{\underline{\alpha}_{1} / 2}, \quad \eta<1, r<1 .
\end{gathered}
$$

Therefore, $V \in \operatorname{WLSC}\left(\underline{\alpha} / 2,0, \sqrt{\underline{c} /\left(2 C_{1}\right)}\right) \cap \operatorname{WUSC}\left(\bar{\alpha} / 2,0, \sqrt{2 \bar{C} C_{1}}\right)$.

Remark 1 The threshold $(0,1)$ in scaling of $V$ in (2.7) may be replaced by any bounded interval at the expense of a constant $\sqrt{2 C_{1} / \underline{c}_{1}}$ (see [5, Section 3]), i.e., for any $R>1$, there is a constant $c$ such that

$$
\frac{V(\eta r)}{V(r)} \leqslant c \eta^{\underline{\alpha}_{1} / 2}, \quad \eta<1, r<R .
$$

We define

$$
M(r)=\frac{V^{2}(r)}{r^{2}}, \quad r>0 .
$$

We note that $M(\cdot)$ is decreasing and $\lim _{r \rightarrow 0^{+}} M(r)=\infty$. To simplify the notation on how the constants depend on the parameters, we put

$$
\sigma=(\bar{\alpha}, \bar{C}, \underline{\alpha}, \underline{c}) \quad \text { and } \quad \underline{\sigma}=\left(\sigma, \underline{\alpha}_{1}, \underline{c}_{1}\right)
$$

Hence, e.g., writing $c=c(\sigma)$, we mean that $c$ depends on $\bar{\alpha}, \bar{C}, \underline{\alpha}, \underline{c}$.

The global weak lower scaling condition (assumption (2.3)) implies $p_{t}(x)$ is jointly continuous on $(0, \infty) \times \mathbb{R}\left(e^{-t \psi} \in L^{1}(\mathbb{R})\right)$ and (see [6, Lemma 1.5])

$$
\begin{gathered}
p_{t}(x) \stackrel{C}{\approx}\left[V^{-1}(\sqrt{t})\right]^{-1} \wedge \frac{t}{V^{2}(|x|)|x|}, \quad t>0, x \in \mathbb{R}, \\
v(x) \stackrel{C}{\approx} \frac{1}{V^{2}(|x|)|x|}, \quad x \neq 0
\end{gathered}
$$


where $C=C(\sigma)$.

Let us denote

$$
p(t, x, y)=p_{t}(y-x) .
$$

By [22, Theorem 1.1 (c)], we have

$$
\left|\partial_{x} p(t, x, y)\right| \leqslant c \frac{1}{V^{-1}(\sqrt{t})} p(t, x, y), \quad t>0, x, y \in \mathbb{R} .
$$

We consider the compensated potential kernel

$$
K(x)=\int_{0}^{\infty}\left(p_{s}(0)-p_{s}(x)\right) \mathrm{d} s, \quad x \in \mathbb{R} .
$$

By symmetry and [1, Theorem II.19], the monotone convergence theorem implies

$$
K(x)=\frac{1}{\pi} \int_{0}^{\infty}(1-\cos x s) \frac{1}{\psi(s)} \mathrm{d} s=\frac{1}{x \pi} \int_{0}^{\infty}(1-\cos s) \frac{1}{\psi(s / x)} \mathrm{d} s, \quad x \neq 0 .
$$

By [21, Proposition 2.2], $K$ is subadditive.

Lemma 2.1 For every $R>0$ there exists a constant $C_{2}=C_{2}(\underline{\sigma}, R \vee 1)$ such that

$$
\left|\partial_{x} K(x)\right| \leqslant C_{2} M(|x| \wedge R), \quad x \in \mathbb{R}
$$

Proof By symmetry we consider only $x \geqslant 0$. Let $r=x \wedge R$. Since $x \mapsto p_{s}(x)$ is non-increasing on $(0, \infty)$, monotonicity of $V(x),(2.9)$ and (2.11) imply

$$
\begin{aligned}
0 \leqslant \partial_{x} K(x) & =\partial_{x} \int_{0}^{\infty} \int_{0}^{x}-\partial_{\rho} p_{s}(\rho) \mathrm{d} \rho \mathrm{d} s=\int_{0}^{\infty}\left|\partial_{x} p_{s}(x)\right| \mathrm{d} s \\
& \leqslant c \int_{0}^{\infty} \frac{1}{V^{-1}(\sqrt{s})^{2}} \wedge \frac{s}{V^{2}(x) x V^{-1}(\sqrt{s})} \mathrm{d} s \\
& \leqslant c \int_{0}^{\infty} \frac{1}{V^{-1}(\sqrt{s})^{2}} \wedge \frac{s}{V^{2}(r) r V^{-1}(\sqrt{s})} \mathrm{d} s \\
& =c \int_{V^{2}(r)}^{\infty} \frac{1}{V^{-1}(\sqrt{s})^{2}} \mathrm{~d} s+\frac{c}{V^{2}(r) r} \int_{0}^{V^{2}(r)} \frac{s}{V^{-1}(\sqrt{s})} \mathrm{d} s .
\end{aligned}
$$


By [5, Remark 4], $V^{-1} \in \operatorname{WLSC}\left(\frac{2}{\bar{\alpha}}, 0, \bar{C}^{-2 / \bar{\alpha}}\right)$, where $1<\bar{\alpha}<2$; hence,

$$
\int_{V^{2}(r)}^{\infty} \frac{1}{V^{-1}(\sqrt{s})^{2}} \mathrm{~d} s \leqslant \frac{c_{1} V(r)^{4 / \bar{\alpha}}}{r^{2}} \int_{V^{2}(r)}^{\infty} \frac{1}{s^{2 / \alpha}} \mathrm{d} s=\frac{c_{1}}{1-\frac{2}{\bar{\alpha}}} \frac{V^{2}(r)}{r^{2}}
$$

where $c_{1}=\bar{C}^{4 / \bar{\alpha}}$. By the explanation in [5, Remark 4] and (2.7), we have

$$
\frac{V^{-1}(\eta t)}{V^{-1}(t)} \geqslant c \eta^{\frac{2}{\underline{\alpha}_{1}}}
$$

for $0<t<1, \eta<1$ and some constant $c=c\left(\underline{\alpha}_{1}, \underline{c}_{1}, C_{1}, V(1)\right)$. This implies

$$
\begin{aligned}
\int_{0}^{V^{2}(r)} \frac{s}{V^{-1}(\sqrt{s})} \mathrm{d} s & =\int_{0}^{V^{2}(r) \wedge 1} \frac{s}{V^{-1}(\sqrt{s})} \mathrm{d} s+\int_{V^{2}(r) \wedge 1}^{V^{2}(r)} \frac{s}{V^{-1}(\sqrt{s})} \mathrm{d} s \\
& \leqslant \frac{c_{2}}{r} \int_{0}^{V^{2}(r) \wedge 1} \frac{V(r)^{2 / \underline{\alpha}_{1}}}{s^{1 / \underline{\alpha}_{1}-1}} \mathrm{~d} s+c_{3} \frac{V^{4}(r)}{2 V^{-1}(1)} \leqslant c_{4}(1+R) \frac{V^{4}(r)}{r} .
\end{aligned}
$$

Hence,

$$
\frac{1}{V^{2}(r) r} \int_{0}^{V^{2}(r)} \frac{s}{V^{-1}(\sqrt{s})} \mathrm{d} s \leqslant c_{4}(1+R) \frac{V^{2}(r)}{r^{2}} .
$$

By [21, Lemma 2.14 with $\left.\underline{\alpha}_{1}\right]$, for $|x| \leqslant R$,

$$
K(x) \approx \frac{V^{2}(|x|)}{|x|}
$$

Hence,

$$
|\partial K(x)| \leqslant M(|x| \wedge R) \approx \frac{K(|x| \wedge R)}{|x| \wedge R} .
$$

Analogously to $\alpha$-stable processes, we define the Kato class for gradient perturbations.

Definition 2 We say that a function $b: \mathbb{R} \rightarrow \mathbb{R}$ belongs to the Kato class $\mathcal{K}_{1}$ if

$$
\lim _{r \rightarrow 0^{+}} \sup _{x \in \mathbb{R}} \int_{B(x, r)} \frac{K(|x-z|)}{|x-z|}|b(z)| \mathrm{d} z=0 .
$$


We note that $L^{\infty}(\mathbb{R}) \subset \mathcal{K}_{1}$. Since $\frac{K(r)}{r} \approx \frac{V^{2}(r)}{r^{2}}$ for small $r>0$, in this paper we will use condition (2.15) in the form

$$
\lim _{r \rightarrow 0^{+}} \sup _{x \in \mathbb{R}} \int_{B(x, r)} \frac{V^{2}(|x-z|)}{|x-z|^{2}}|b(z)| \mathrm{d} z=0 .
$$

We consider the time-homogeneous transition probabilities

$$
P_{t}(x, A)=\int_{A} p(t, x, y) \mathrm{d} y, \quad t>0, x \in \mathbb{R}, A \subset \mathbb{R} .
$$

By Kolmogorov's and Dynkin-Kinney's theorems the transition probability $P_{t}$ defines in the usual way Markov probability measures $\left\{\mathbb{P}^{x}, x \in \mathbb{R}\right\}$ on the space $\Omega$ of the rightcontinuous and left-limited functions $\omega:[0, \infty) \rightarrow \mathbb{R}$. We let $\mathbb{E}^{x}$ be the corresponding expectations. We will denote by $X=\left\{X_{t}\right\}_{t} \geqslant 0$ the canonical process on $\Omega, X_{t}(\omega)=$ $\omega(t)$. Hence,

$$
\mathbb{P}\left(X_{t} \in B\right)=\int_{B} p(t, x, y) \mathrm{d} y .
$$

For any open set $D$, we define the first exit time of the process $X_{t}$ from $D$,

$$
\tau_{D}=\inf \left\{t>0: X_{t} \notin D\right\}
$$

Now, by the usual Hunt's formula, we define the transition density of the process killed when leaving $D[2,6,15]$ :

$$
p_{D}(t, x, y)=p(t, x, y)-\mathbb{E}^{x}\left[\tau_{D}<t ; p\left(t-\tau_{D}, X_{\tau_{D}}, y\right)\right], \quad t>0, x, y \in \mathbb{R} .
$$

We briefly recall some well-known properties of $p_{D}$ (see [6]). The function $p_{D}$ satisfies the Chapman-Kolmogorov equations

$$
\int_{\mathbb{R}} p_{D}(s, x, z) p_{D}(t, z, y) \mathrm{d} z=p_{D}(s+t, x, y), \quad s, t>0, x, y \in \mathbb{R} .
$$

Furthermore, $p_{D}$ is jointly continuous when $t \neq 0$, and we have

$$
0 \leqslant p_{D}(t, x, y)=p_{D}(t, y, x) \leqslant p(t, x, y) .
$$

In particular,

$$
\int_{\mathbb{R}} p_{D}(t, x, y) \mathrm{d} y \leqslant 1 .
$$

If $D$ is a $C^{1,1}$ domain (see definition in Sect. 3), by Blumenthal's zero-one law, symmetry of $p_{t}$, we have $\mathbb{P}^{x}\left(\tau_{D}=0\right)=1$ for every $x \in D^{c}$. In particular, $p_{D}(t, x, y)=0$ if $x \in D^{c}$ or $y \in D^{c}$. 


\section{Green Function of $\mathcal{L}$}

We define the Green function of $X_{t}$ for $D$,

$$
G_{D}(x, y)=\int_{0}^{\infty} p_{D}(t, x, y) \mathrm{d} t, \quad x, y \in \mathbb{R}
$$

and the Green operator

$$
G_{D} \phi(x)=\int_{\mathbb{R}} G_{D}(x, y) \phi(y) \mathrm{d} y, \quad x \in \mathbb{R} .
$$

From now on, every time we mention the Green function, it should be understood as the Green function of $D$, and then $G=G_{D}$.

Definition 3 We say that a function $f: \mathbb{R} \rightarrow \mathbb{R}$ is a $\mathcal{L}$-harmonic (or simply harmonic) function on an open bounded set $D \subset \mathbb{R}$ if for any open $F \subset \bar{F} \subset D$ and $x \in F$

$$
f(x)=\mathbb{E}^{x} f\left(X_{\tau_{F}}\right) .
$$

We say that a function $f$ is a regular $\mathcal{L}$-harmonic (or simply regular harmonic) function on an open bounded set $D \subset \mathbb{R}$ if for every $x \in D$

$$
f(x)=\mathbb{E}^{x} f\left(X_{\tau_{D}}\right) .
$$

Note that for fixed $x \in D$ the function $G(x, \cdot)$ is harmonic on $D \backslash\{x\}$ and regularly harmonic on $D \backslash \overline{B(x, \varepsilon)}$, where $B(x, \varepsilon) \subset D$. By [36, Theorem 1.1], we know that the function $K(x)$ is harmonic on $\mathbb{R} \backslash\{0\}$.

Definition 4 We call a set $D \subset \mathbb{R}$ a $C^{1,1}$ open set at scale $r>0$ if it is an union of open intervals of length at least $r$ and distanced one from another at least $r$. The number $r_{0}=\sup \{r: D$ is at scale $r\}$ is called the localization radius.

Definition 4 corresponds to the definition of multidimensional $C^{1,1}$ set with localization radius $r_{0}$. In what follows, we assume that

$$
D \text { is a } C^{1,1} \text { set with } \operatorname{diam}(D)<\infty \text { and localization radius } r_{0}=r_{0}(D) \text {. }
$$

Some constants will depend on the ratio $\operatorname{diam}(D) / r_{0}$ called the distortion of the set $D$.

Lemma 3.1 There exists a constant $C_{3}=C_{3}\left(\underline{\sigma}, \operatorname{diam}(D) / r_{0}, 1 \vee \operatorname{diam}(D)\right)$ such that

$$
G(x, y) \stackrel{C_{3}}{\approx} V\left(\delta_{x}\right) V\left(\delta_{y}\right)\left(\frac{1}{\sqrt{\delta_{x} \delta_{y}}} \wedge \frac{1}{|x-y|}\right), \quad x, y \in D
$$


Proof Note that (see [6, Proposition 4.4 and Theorem 4.5])

$p_{D}(t, x, y) \approx e^{-2 \gamma(D) t}\left(\frac{V\left(\delta_{x}\right)}{\sqrt{t / 2} \wedge V\left(r_{0}\right)} \wedge 1\right)\left(\frac{V\left(\delta_{y}\right)}{\sqrt{t / 2} \wedge V\left(r_{0}\right)} \wedge 1\right) p\left(t \wedge V^{2}\left(r_{0}\right), x, y\right)$

where $\frac{1}{8}\left(\operatorname{diam}(D) / r_{0}\right)^{2} \leqslant \gamma(D) V^{2}\left(r_{0}\right) \leqslant c\left(\operatorname{diam}(D) / r_{0}\right)^{1 / 2}$. Now, integrating them against time, we get

$$
\begin{aligned}
G(x, y) \stackrel{c_{1}}{\approx}\left(V\left(\delta_{x}\right) \wedge V\left(r_{0}\right)\right)\left(V\left(\delta_{y}\right) \wedge V\left(r_{0}\right)\right) p\left(V^{2}\left(r_{0}\right), x, y\right) \\
+\int_{0}^{V^{2}\left(r_{0}\right)}\left(\frac{V\left(\delta_{x}\right)}{\sqrt{t}} \wedge 1\right)\left(\frac{V\left(\delta_{y}\right)}{\sqrt{t}} \wedge 1\right) p(t, x, y) d t
\end{aligned}
$$

where the comparability constant $c_{1}$ depends on the scaling characteristics in (2.6) and (2.7) and the distortion of $D$. Now, by the same calculation as in the proof of [13, Theorem 7.3 (iii) and Corollary 7.4], we obtain

$$
G(x, y) \stackrel{c_{2}}{\approx} V\left(\delta_{x}\right) V\left(\delta_{y}\right)\left(\frac{1}{V^{-1}\left(\sqrt{V\left(\delta_{x}\right) V\left(\delta_{y}\right)}\right)} \wedge \frac{1}{|x-y|}\right),
$$

where the comparability constant $c_{2}$ depends on the scaling characteristics in (2.6) and (2.7), the distortion of $D$ and $1 \vee \operatorname{diam}(D)$.

Let us consider $x, y \in D$ such that $\frac{1}{V^{-1}\left(\sqrt{V\left(\delta_{x}\right) V\left(\delta_{y}\right)}\right)}<\frac{1}{|x-y|}$; this means

$$
V^{2}(|x-y|)<V\left(\delta_{x}\right) V\left(\delta_{y}\right)
$$

Without loss of generality we may and do assume $\delta_{x} \leqslant \delta_{y}$. Then,

$$
\begin{aligned}
V^{2}(|x-y|) & <V\left(\delta_{x}\right) V\left(\delta_{x}+\left(\delta_{y}-\delta_{x}\right)\right) \leqslant V\left(\delta_{x}\right) V\left(\delta_{x}+|x-y|\right) \\
& \leqslant V\left(\delta_{x}\right)\left[V\left(\delta_{x}\right)+V(|x-y|)\right]
\end{aligned}
$$

which implies $V(|x-y|) \leqslant 2 V\left(\delta_{x}\right)$. By monotonicity and subadditivity of $V$ we obtain that

$$
V\left(\delta_{x}\right) \leqslant V\left(\delta_{y}\right) \leqslant V(|x-y|)+V\left(\delta_{x}\right) \leqslant 3 V\left(\delta_{x}\right) .
$$

As a consequence of (2.6), we obtain

$$
\delta_{y} \leqslant\left(18 C_{1} / \underline{\alpha}\right)^{1 /(2 \underline{\alpha})} \delta_{x} .
$$

Again, by (2.6), we get

$$
V^{-1}\left(\sqrt{V\left(\delta_{x}\right) V\left(\delta_{y}\right)}\right) \stackrel{c_{3}}{\approx} \sqrt{\delta_{x} \delta_{y}}
$$


where $c_{3}=c_{3}(\underline{\alpha}, \underline{c})$. Now, let

$$
V^{2}(|x-y|) \geqslant V\left(\delta_{x}\right) V\left(\delta_{y}\right)
$$

We only need to show that $2|x-y|^{2} \geqslant \delta_{x} \delta_{y}$. Without loss of generality we can and do assume $\delta_{x} \leqslant \delta_{y}$. By monotonicity of $V,|x-y| \geqslant \delta_{x}$. Hence, $\delta_{y} \leqslant|x-y|+\delta_{x} \leqslant$ $2|x-y|$, which completes the proof.

\subsection{Estimates of the Poisson Kernel}

If $D$ is $C^{1,1}$, it is known that the harmonic measure of $D$ has a density and we call it the Poisson kernel. By the Ikeda-Watanabe formula [23] it is equal to

$$
P_{D}(x, z)=\int_{D} G(x, y) v(z-y) \mathrm{d} y, \quad x \in D, z \in \bar{D}^{c} .
$$

First, we give the estimates of the Poisson kernel in the special case $D=B(0, R)$.

Lemma 3.2 Let $R>0$. Suppose $D=B(0, R)$. Then,

$$
P_{D}(x, z) \stackrel{C_{4}}{\approx} \frac{V\left(\delta_{x}\right)}{V\left(\delta_{z}\right)|x-z|}\left(\frac{V(R)}{V\left(\delta_{z}\right)} \wedge 1\right), \quad x \in D, z \in \bar{D}^{c}
$$

where $C_{4}=C_{4}(\underline{\sigma}, 1 \vee R)>0$.

Proof To distinguish this case from the general one considered in Proposition 3.3 below, we denote $B=B(0, R)$. Then, for $x \in \mathbb{R}, \delta_{x}=\delta_{x}^{B}$. By (3.7), Lemma 3.1 and (2.10), there is $c_{1}=c_{1}(\underline{\sigma}, 1 \vee R)$ such that

$$
\begin{aligned}
P_{B}(x, z) & =\int_{B} G_{B}(x, y) v(|y-z|) \mathrm{d} z \\
& \stackrel{c_{1}}{\approx} \int_{B} V\left(\delta_{x}\right) V\left(\delta_{y}\right)\left(\frac{1}{\sqrt{\delta_{x} \delta_{y}}} \wedge \frac{1}{|x-y|}\right) \frac{\mathrm{d} y}{V^{2}(|z-y|)|z-y|} .
\end{aligned}
$$

By Remark 1, we obtain inequality (2.7) for $r<3 R$ with constant $c_{2}=$ $c_{2}\left(\underline{\alpha}_{1}, \underline{c}_{1}, 1 \vee R\right)$. Hence, for $|z|<2 R$, we have

$$
\begin{aligned}
& \left(2 \bar{C} C_{1}\right)^{-1 / 2}\left(\frac{\delta_{z}}{|z-y|}\right)^{\bar{\alpha} / 2} \leqslant \frac{V\left(\delta_{z}\right)}{V(|z-y|)} \leqslant c_{2}\left(\frac{\delta_{z}}{|z-y|}\right)^{\underline{\alpha}_{1} / 2} \\
& \left(2 \bar{C} C_{1}\right)^{-1 / 2}\left(\frac{\delta_{y}}{|z-y|}\right)^{\bar{\alpha} / 2} \leqslant \frac{V\left(\delta_{y}\right)}{V(|z-y|)} \leqslant c_{2}\left(\frac{\delta_{y}}{|z-y|}\right)^{\underline{\alpha}_{1} / 2} .
\end{aligned}
$$


These imply

$$
\begin{aligned}
P_{B}(x, z) & \leqslant c_{1} c_{2}^{2} \int_{B} \frac{V\left(\delta_{x}\right)}{V\left(\delta_{z}\right)}\left(\frac{1}{\sqrt{\delta_{x} \delta_{y}}} \wedge \frac{1}{|x-y|}\right) \frac{\left(\delta_{y} \delta_{z}\right)^{\underline{\alpha}_{1} / 2}}{|z-y|^{1+\underline{\alpha}_{1}}} \mathrm{~d} y \\
& \stackrel{c_{3}}{\approx} \int_{B} \frac{V\left(\delta_{x}\right)}{V\left(\delta_{z}\right)}\left(\frac{\delta_{z}}{\delta_{x}}\right)^{\underline{\alpha}_{1} / 2} G_{B}^{S \underline{\alpha}_{1} S}(x, y) \frac{\mathrm{d} y}{|z-y|^{1+\underline{\alpha}_{1}}} \\
& \approx \frac{V\left(\delta_{x}\right)}{V\left(\delta_{z}\right)}\left(\frac{\delta_{z}}{\delta_{x}}\right)^{\underline{\alpha}_{1} / 2} P_{B}^{S_{\alpha_{1}} S}(x, z) .
\end{aligned}
$$

Here, $c_{3}=c_{3}\left(c_{1}, c_{2}, \underline{\alpha}_{1}\right)$ and $S \underline{\alpha}_{1} S$ refers to the symmetric $\alpha$-stable process with index of stability $\underline{\alpha}_{1}$. Similarly, we obtain

$$
P_{B}(x, z) \geqslant c_{4} \frac{V\left(\delta_{x}\right)}{V\left(\delta_{z}\right)}\left(\frac{\delta_{z}}{\delta_{x}}\right)^{\bar{\alpha} / 2} P_{B}^{S \alpha \bar{\alpha} S}(x, z),
$$

where $c_{4}=c_{4}\left(c_{1}, \bar{\alpha}, \bar{C}\right)$. By formula for $P_{B}^{S \underline{\alpha}_{1} S}(x, z)$ [3, Theorem A], we get the assertion of the lemma for $|z|<2 R$.

If $|z| \geqslant 2 R$, by (2.5) and [20, Proposition 3.5], we get

$$
P_{B}(x, z) \approx v(|z|) \mathbb{E}^{x} \tau_{B} \approx V\left(\delta_{x}\right) V(R) v(|z|),
$$

which implies the claim of the lemma.

Proposition 3.3 There exists a constant $C_{5}=C_{5}\left(\underline{\sigma}\right.$, diam(D) $\left./ r_{0}, 1 \vee \operatorname{diam}(\mathrm{D})\right)$ such that

$$
P_{D}(x, z) \stackrel{C_{5}}{\approx} \frac{V\left(\delta_{x}\right)}{V\left(\delta_{z}\right)|x-z|}\left(\frac{V(\operatorname{diam}(\mathrm{D}))}{V\left(\delta_{z}\right)} \wedge 1\right), \quad x \in D, z \in D^{c} .
$$

Proof Let $x \in D, z \in D^{c}$. By Lemma 3.2 we consider only the case when $D$ is a union of at least two open intervals. Let $B$ be an open interval such that $x \in B$ and $\tilde{D}=D \backslash B$ is open. By the Ikeda-Watanabe formula

$$
P_{D}(x, z)=\int_{B} G(x, y) v(|y-z|) \mathrm{d} y+\int_{\tilde{D}} G(x, y) v(|y-z|) \mathrm{d} y=: \mathrm{I}+\mathrm{II} .
$$

Lemma 3.1 implies

$$
G(x, y) \stackrel{c_{1}}{\approx} G_{B}(x, y), \quad x, y \in B
$$

for $c_{1}=c_{1}\left(C_{3}\right)$. Hence, by Lemma 3.2

$$
\mathrm{I} \stackrel{c_{1}}{\approx} \int_{B} G_{B}(x, y) v(|y-z|) \mathrm{d} y \stackrel{c_{2}}{\approx} \frac{V\left(\delta_{x}\right)}{V(\operatorname{dist}(z, B))|x-z|}\left(\frac{V(\operatorname{diam}(B))}{V\left(\delta_{z}\right)} \wedge 1\right)
$$


where $c_{2}=c_{2}\left(C_{3}, C_{4}\right)$. If $\operatorname{dist}(z, B)=\delta_{z}$, the lower bound follows by (3.9). Suppose $\operatorname{dist}(z, B)>\delta_{z}$, and let $\tilde{B}$ be a connected component of $\tilde{D} \operatorname{such}$ that $\operatorname{dist}(z, \tilde{B})=\delta_{z}$. Note that $r_{0} \leqslant \operatorname{dist}(x, \tilde{B}) \leqslant|x-z|+\operatorname{dist}(z, \tilde{B}) \leqslant 2|x-z|$. Therefore, by Lemma 3.1 ,

$\mathrm{II} \geqslant \frac{1}{C_{3}} \int_{\tilde{B}} \frac{V\left(\delta_{x}\right) V\left(\delta_{y}\right)}{\operatorname{diam}(D)} v(|y-z|) \mathrm{d} y \geqslant \frac{r_{0}}{2 C_{3} \operatorname{diam}(D)} \frac{V\left(\delta_{x}\right)}{|x-z|} \int_{\tilde{B}} V\left(\delta_{y}\right) v(|y-z|) \mathrm{d} y$.

Now, (2.10) and (2.5) imply

$$
\begin{aligned}
\int_{\tilde{B}} V\left(\delta_{y}\right) v(|y-z|) \mathrm{d} y & \geqslant c_{3} \int_{\tilde{B}} \frac{V\left(\delta_{y}\right)}{V^{2}(|z-y|)|z-y|} \mathrm{d} y \\
& \geqslant c_{3} \int_{0}^{\delta_{z} \wedge r_{0} / 2} \frac{V(s)}{V^{2}\left(s+\delta_{z}\right)\left(s+\delta_{z}\right)} \mathrm{d} s \\
& \geqslant \frac{c_{3}}{V^{2}\left(2 \delta_{z}\right) 2 \delta_{z}} \int_{0}^{\delta_{z} \wedge r_{0} / 2} V(s) \mathrm{d} s \\
& \geqslant \frac{c_{4}}{V^{2}\left(2 \delta_{z}\right) \delta_{z}} \int_{\left(\delta_{z} \wedge r_{0} / 2\right) / 2}^{\delta_{z} \wedge r_{0} / 2} V(s) \mathrm{d} s \\
& \geqslant \frac{c_{4}}{4} \frac{\left(\delta_{z} \wedge r_{0} / 2\right) V\left(\delta_{z} \wedge r_{0} / 2\right)}{V^{2}\left(2 \delta_{z}\right) \delta_{z}}
\end{aligned}
$$

Hence, we obtain the lower bound in this case.

Next, we will prove the upper bound for the second integral. Let $\lambda=\delta_{z} \wedge \operatorname{diam}(D)$ and $D_{1}=\tilde{D} \cap\left\{y: \delta_{y} \leqslant \lambda\right\}$ and $D_{2}=\tilde{D} \cap\left\{y: \delta_{y}>\lambda\right\}$. By weak scaling conditions, we obtain

$$
\begin{aligned}
\mathrm{II} & \stackrel{c_{5}}{\approx} \int_{\tilde{D}} \frac{V\left(\delta_{x}\right) V\left(\delta_{y}\right)}{|x-y|} \frac{\mathrm{d} y}{V^{2}(|z-y|)|z-y|} \leqslant \frac{V\left(\delta_{x}\right)}{r_{0}} \int_{\tilde{D}} \frac{V\left(\delta_{y}\right) \mathrm{d} y}{V^{2}(|z-y|)|z-y|} \\
& \leqslant \frac{V\left(\delta_{x}\right)}{r_{0}}\left(\frac{\left|D_{1}\right| V(\lambda)}{V^{2}\left(\delta_{z}\right) \delta_{z}}+\int_{D_{2}} \frac{\mathrm{d} y}{V\left(\delta_{y}\right) \delta_{y}}\right) \\
& \leqslant \frac{V\left(\delta_{x}\right)}{r_{0}}\left(\frac{2 \operatorname{diam}(D)}{r_{0}} \frac{\lambda V(\lambda)}{V^{2}\left(\delta_{z}\right) \delta_{z}}+c_{6} \frac{\mathbf{1}_{\lambda=\delta_{z}}}{V(\lambda)}\right) \\
& \approx \frac{V\left(\delta_{x}\right)}{V\left(\delta_{z}\right)|x-z|}\left(\frac{V(\operatorname{diam} D)}{V\left(\delta_{z}\right)} \wedge 1\right) .
\end{aligned}
$$

where $c_{5}=c_{5}\left(C_{3}, C\right)$ and the constant $c_{6}$ depends only on the scaling characteristics. This completes the proof. 


\subsection{Estimates of $\partial_{x} G(x, y)$}

Below, we will prove various estimates of $\partial_{x} G(x, y)$ according to the range of variables $x$ and $y$. We summarize these results in Theorem 3.10. First, we will need the following auxiliary lemma.

Lemma 3.4 Let $x \in D$. There is a constant $C_{6}=C_{6}\left(\underline{\sigma}, \operatorname{diam}(\mathrm{D}) / r_{0}, 1 \vee \operatorname{diam}(\mathrm{D})\right)$ such that

$$
\int_{\mathbb{R}} \frac{M(|x-z|)}{V\left(\delta_{z}\right)} \mathrm{d} z \leqslant C_{6} \frac{V\left(\delta_{x}\right)}{\delta_{x}} .
$$

Proof Note that for $r<R$, by (2.8), we have

$$
\int_{0}^{r} M(s) \mathrm{d} s=\int_{0}^{r} \frac{V^{2}(s)}{s^{2}} \mathrm{~d} s \leqslant c_{1} \int_{0}^{r} \frac{V^{2}(r) s^{\underline{\alpha}_{1}-2}}{r^{\underline{\alpha}} \underline{1}} \mathrm{~d} s=c_{2} \frac{V^{2}(r)}{r},
$$

with $c_{2}$ depending on $R$. Let $B_{1}=B\left(x, \delta_{x} / 2\right)$. By symmetry of $M,(2.5)$ and (3.10), we have

$$
\int_{B_{1}} \frac{M(|x-z|)}{V\left(\delta_{z}\right)} \mathrm{d} z \leqslant 2 \int_{0}^{\delta_{x} / 2} \frac{M(s)}{V\left(\delta_{x} / 2\right)} \mathrm{d} s \leqslant 2 c_{2} \frac{V\left(\delta_{x} / 2\right)}{\delta_{x}} \leqslant 2 c_{2} \frac{V\left(\delta_{x}\right)}{\delta_{x}} .
$$

Note that for $z \notin B_{1}$, we have $\delta_{z} \leqslant 3|x-z|$ and $\delta_{x} \leqslant 2|x-z|$. Hence, by (2.6),

$$
\begin{aligned}
\int_{B_{1}^{c} \cap\left\{\delta_{x} \leqslant \delta_{z}\right\}} \frac{M(|x-z|)}{V\left(\delta_{z}\right)} \mathrm{d} z & \leqslant c_{3} \int_{\left\{\delta_{x} \leqslant \delta_{z}\right\}} \frac{V\left(\delta_{z}\right)}{\delta_{z}^{2}} \mathrm{~d} z \leqslant c_{4} \int_{\left\{\delta_{x} \leqslant \delta_{z}\right\}} \frac{V\left(\delta_{x}\right) \delta_{z}^{\bar{\alpha} / 2-2}}{\delta_{x}^{\bar{\alpha} / 2}} \mathrm{~d} z \\
& \leqslant c_{4} \sum_{k=1}^{2 n} \int_{B\left(a_{k}, \delta_{x}\right)^{c}} \frac{V\left(\delta_{x}\right)|s|^{\bar{\alpha} / 2-2}}{\delta_{x}^{\bar{\alpha} / 2}} \mathrm{~d} s=c_{5} \frac{V\left(\delta_{x}\right)}{\delta_{x}},
\end{aligned}
$$

where $D=\bigcup_{k=1}^{n}\left(a_{2 k-1}, a_{2 k}\right)$. Similarly,

$$
\begin{aligned}
\int_{B_{1}^{c} \cap\left\{\delta_{z}<\delta_{x}\right\}} \frac{M(|x-z|)}{V\left(\delta_{z}\right)} \mathrm{d} z & \leqslant c_{6} \int_{\left\{\delta_{z}<\delta_{x}\right\}} \frac{V^{2}\left(\delta_{x}\right)}{\delta_{x}^{2} V\left(\delta_{z}\right)} \mathrm{d} z \leqslant c_{7} \int_{\left\{\delta_{z}<\delta_{x}\right\}} \frac{V\left(\delta_{x}\right)}{\delta_{x}^{2-\bar{\alpha} / 2} \delta_{z}^{\bar{\alpha} / 2}} \mathrm{~d} z \\
& \leqslant c_{7} \sum_{k=1}^{2 n} \int_{B\left(a_{k}, \delta_{x}\right)} \frac{V\left(\delta_{x}\right)}{\delta_{x}^{2-\bar{\alpha}} s^{\bar{\alpha} / 2}} \mathrm{~d} s=c_{8} \frac{V\left(\delta_{x}\right)}{\delta_{x}}
\end{aligned}
$$

Proposition 3.5 There is a constant $C_{7}=C_{7}\left(\underline{\sigma}\right.$, diam(D) $\left./ r_{0}, 1 \vee \operatorname{diam}(\mathrm{D})\right)$ such that

$$
\left|\partial_{x} G(x, y)\right| \leqslant C_{7}\left(M(|x-y|)+\frac{G(x, y)}{\delta_{x}} \mathbb{1}_{\frac{|x-y|}{2}>\delta_{x}}\right) .
$$


Proof Since $X_{t}$ is translation invariant, we may and do assume that $0 \notin D$. Let $x, y \in D$ and $x \neq y$. It is known (see [21, Lemma 2.3])

$$
G_{\{0\}^{c}}(x, y)=K(x)+K(y)-K(y-x) .
$$

Hence, by symmetry,

$$
\begin{aligned}
G(x, y) & =G_{\{0\}^{c}}(x, y)-\mathbb{E}^{y} G_{\{0\}^{c}}\left(x, X_{\tau_{D}}\right) \\
& =K(y)-K(x-y)-\mathbb{E}^{y} K\left(X_{\tau_{D}}\right)+\mathbb{E}^{y} K\left(x-X_{\tau_{D}}\right) .
\end{aligned}
$$

By Lemma 2.1 and the dominated convergence theorem,

$$
\partial_{x} G(x, y)=\mathbb{E}^{y} \partial_{x} K\left(x-X_{\tau_{D}}\right)-\partial_{x} K(x-y)
$$

Again, by Lemma 2.1 and (2.14), for $|x-z| \geqslant|x-y| / 2$, we have

$$
\left|\partial_{x} K(x-z)\right| \leqslant c_{1} M(|x-z| \wedge \operatorname{diam}(D)) \leqslant c_{2} M(|x-y|) .
$$

This implies

$$
\begin{aligned}
\left|\partial_{x} G(x, y)\right| & \leqslant c_{3} M(|x-y|)+\mathbb{E}^{y}\left|\partial_{x} K\left(x-X_{\tau_{D}}\right)\right| \\
& \leqslant c_{4} M(|x-y|)+\mathbb{E}^{y}\left[\left|\partial_{x} K\left(x-X_{\tau_{D}}\right)\right|,\left|x-X_{\tau_{D}}\right| \leqslant \frac{|x-y|}{2}\right] .
\end{aligned}
$$

It remains to estimate

$$
I:=\mathbb{E}^{y}\left[\left|\partial_{x} K\left(x-X_{\tau_{D}}\right)\right|,\left|x-X_{\tau_{D}}\right| \leqslant \frac{|x-y|}{2}\right] .
$$

If $\delta_{x} \geqslant \frac{|x-y|}{2}, I=0$. So let $\delta_{x}<\frac{|x-y|}{2}$. Note that if $|x-z| \leqslant|x-y| / 2$, then $|y-z| \geqslant|x-y| / 2$, and in consequence, by Proposition 3.3,

$$
P_{D}(y, z) \lesssim \frac{V\left(\delta_{y}\right)}{V\left(\delta_{z}\right)} \frac{1}{|y-z|} \leqslant \frac{V\left(\delta_{y}\right)}{V\left(\delta_{z}\right)} \frac{2}{|x-y|} .
$$

By Lemma 3.4,

$$
\begin{aligned}
I \leqslant \int_{D^{c} \cap B\left(x, \frac{|x-y|}{2}\right)} M(|x-z|) P_{D}(y, z) \mathrm{d} z & \leqslant c_{5} \frac{V\left(\delta_{y}\right)}{|x-y|} \int_{\mathbb{R}} M(|x-z|) \frac{\mathrm{d} z}{V\left(\delta_{z}\right)} \\
& \leqslant c_{6} \frac{V\left(\delta_{y}\right)}{|x-y|} \frac{V\left(\delta_{x}\right)}{\delta_{x}} .
\end{aligned}
$$

Since $\delta_{x} \leqslant \frac{|x-y|}{2}$, we have $\delta_{y} \leqslant \frac{3}{2}|x-y|$ and by Lemma 3.1,

$$
\frac{V\left(\delta_{y}\right)}{|x-y|} \frac{V\left(\delta_{x}\right)}{\delta_{x}} \lesssim \frac{G(x, y)}{\delta_{x}} .
$$


Hence,

$$
\left|\partial_{x} G(x, y)\right| \lesssim M(|x-y|)+\frac{G(x, y)}{\delta_{x}} \mathbb{1}_{\frac{|x-y|}{2}>\delta_{x}},
$$

which ends the proof.

By Lemma 3.1 and Proposition 3.5, we get a weaker but also useful estimate.

Corollary 3.6 There is a constant $C_{8}=C_{8}(\underline{\sigma}, \operatorname{diam}(D) \vee 1)$ such that

$$
\left|\partial_{x} G(x, y)\right| \leqslant C_{8} M\left(\delta_{x} \wedge|x-y|\right)
$$

Lemma 3.7 If $f \in \mathcal{K}_{1}$, then

$$
\partial_{y} \int_{D} G(y, z) f(z) \mathrm{d} z=\int_{D} \partial_{y} G(y, z) f(z) \mathrm{d} z, \quad y \in D .
$$

Proof Let $0<h<\delta_{y} / 2$. Then, by Corollary 3.6 and monotonicity of $M$,

$$
\begin{aligned}
& \left|\frac{G(y+h, z)-G(y, z)}{h}\right|=\frac{1}{h}\left|\int_{0}^{1} \partial_{s} G(y+s h, z) \mathrm{d} s\right| \\
& =\left|\int_{0}^{1} \partial_{y} G(y+s h, z) \mathrm{d} s\right| \leqslant C_{8} \int_{0}^{1}\left(M\left(\delta_{y+s h} \wedge|y+s h-z|\right)\right) \mathrm{d} s \\
& \quad \leqslant C_{8} \int_{0}^{1}\left(M\left(\delta_{y} / 2\right)+M(|y+s h-z|)\right) \mathrm{d} s \\
& =C_{8}\left(M\left(\delta_{y} / 2\right)+\int_{0}^{1} M(|y+s h-z|) \mathrm{d} s\right) .
\end{aligned}
$$

We only need to prove the uniform integrability of $\int_{0}^{1} M(|y+s h-z|) \mathrm{d} s$ in $h$ on $D$ against $|f(z)| \mathrm{d} z$. If $|h|<\frac{|y-z|}{2}$, by monotonicity $M$ and (2.8), we have

$$
\int_{0}^{1} M(|y+s h-z|) \mathrm{d} s \leqslant M(|y-z| / 2) \leqslant 4 M(|y-z|) .
$$

Next, if $|h| \geqslant \frac{|y-z|}{2}$, by monotonicity and symmetry of $M,(3.10)$ and (2.8), we have

$$
\begin{aligned}
\int_{0}^{1} M(|y+s h-z|) \mathrm{d} s & \leqslant \int_{-1}^{1} M(|y-z+s h|) \mathrm{d} s \leqslant \int_{-1}^{1} M(|s h|) \mathrm{d} s \\
& =2 \int_{0}^{1} M(|s h|) \mathrm{d} s=\frac{2}{|h|} \int_{0}^{|h|} M(s) \mathrm{d} s \leqslant c M(|h|) \\
& \leqslant 4 c M(|y-z|) .
\end{aligned}
$$

Since $f \in \mathcal{K}_{1}$ and (2.15) holds, we obtain the assertion of the lemma. 
Proposition 3.8 Let $x \in D, 0<\varepsilon<\delta_{x}, B=B(x, \varepsilon)$ and $A=B^{c} \cap D$. Then,

$$
\partial_{x} G(x, y)=\int_{B} \partial_{x} G(x, z) P_{A}(y, z) \mathrm{d} z
$$

Proof Fix $x \in D$. Then, $G(x, \cdot)$ is regular harmonic on $A=D \cap[x-\varepsilon, x+\varepsilon]^{c}$ for every $0<\varepsilon<\delta_{x}$. This means

$$
G(x, y)=\mathbb{E}^{y} G\left(x, X_{\tau_{A}}\right)=\int_{B} G(x, z) P_{A}(y, z) \mathrm{d} z, \quad y \in A .
$$

Let us fix $y \in A$. For $z \in B$, we define $P_{1}(y, z)=P_{A}(y, z) \mathbf{1}_{B(x, \varepsilon / 2)}(z)$ and $P_{2}(y, z)=$ $P_{A}(y, z)-P_{1}(y, z)$. Since $P_{1}$ is bounded, we have $P_{1}(y, \cdot) \in \mathcal{K}_{1}$ and by Lemma 3.7,

$$
\partial_{x} \int_{B} G(x, z) P_{1}(y, z) \mathrm{d} z=\int_{B} \partial_{x} G(x, z) P_{1}(y, z) \mathrm{d} z .
$$

Since $\partial_{x} G(x, z)$ is finite on the support of $P_{2}(y, \cdot)$, by the mean value theorem and the dominated convergence theorem, we get

$$
\begin{aligned}
& \lim _{h \rightarrow 0} \frac{\int_{B} G(x+h, z) P_{2}(y, z) \mathrm{d} z-\int_{B} G(x, z) P_{2}(y, z) \mathrm{d} z}{h} \\
& \quad=\lim _{h \rightarrow 0} \int_{B} \frac{G(x+h, z)-G(x, z)}{h} P_{2}(y, z) \mathrm{d} z=\int_{B} \partial_{x} G(x, z) P_{2}(y, z) \mathrm{d} z .
\end{aligned}
$$

These imply

$$
\begin{aligned}
\partial_{x} \int_{B} G(x, z) P_{A}(y, z) \mathrm{d} z & =\partial_{x} \int_{B} G(x, z) P_{1}(y, z) \mathrm{d} z+\partial_{x} \int_{B} G(x, z) P_{2}(y, z) \mathrm{d} z \\
& =\int_{B} \partial_{x} G(x, z) P_{1}(y, z) \mathrm{d} z+\int_{B} \partial_{x} G(x, z) P_{2}(y, z) \mathrm{d} z \\
& =\int_{B} \partial_{x} G(x, z) P_{A}(y, z) \mathrm{d} z,
\end{aligned}
$$

which completes the proof.

Lemma 3.9 Let $x, y \in D$ and $\delta_{x}<2|x-y|$. Then, there exists a constant $C_{9}=$ $C_{9}\left(\underline{\sigma}, \operatorname{diam}(\mathrm{D}) / r_{0}, 1 \vee \operatorname{diam}(\mathrm{D})\right)$ such that

$$
\left|\partial_{x} G(x, y)\right| \leqslant C_{9} \frac{G(x, y)}{\delta_{x}} .
$$


Proof Let $B \subset D$ be any interval such that $\bar{B} \subset D$, and put $A=B^{c} \cap D$. For any $x \in B$ and $y \in D$ such that $x \neq y$, by Propositions 3.5 and 3.8 and harmonicity of $G$,

$$
\begin{aligned}
\left|\partial_{x} G(x, y)\right| & =\left|\int_{B} \partial_{x} G(x, z) P_{A}(y, z) \mathrm{d} z\right| \\
& \leqslant C_{7} \int_{B}\left(M(|x-z|)+\frac{G(x, z)}{\delta_{x}} \mathbb{1}_{\frac{|x-z|}{2}>\delta_{x}}\right) P_{A}(y, z) \mathrm{d} z \\
& \leqslant C_{7} \int_{B} M(|x-z|) P_{A}(y, z) \mathrm{d} z+C_{7} \frac{G(x, y)}{\delta_{x}} .
\end{aligned}
$$

Therefore, it remains to estimate the integral

$$
\int_{B} M(|x-z|) P_{A}(y, z) \mathrm{d} z
$$

Let $B=B\left(x, \delta_{x} / 4\right)$. By the assumption, $y \notin B$, $\operatorname{dist}(y, B) \geqslant \delta_{x} / 4$ and $|y-z| \approx$ $|x-y|$ for $z \in B$.

Note that $4 \delta_{x}^{A}=\delta_{x}$ and $\delta_{y}^{A} \leqslant \delta_{y}$. If $\delta_{y}^{A}<\delta_{y}$ then $\delta_{y}^{A}=\delta_{y}^{B} \geqslant \delta_{x} / 4$ and consequently $\delta_{y} \leqslant \delta_{y}^{A}+\sup _{z \in B} \delta_{z} \leqslant \delta_{y}^{A}+5 \delta_{x} / 4 \leqslant 6 \delta_{y}^{A}$. Hence, $\delta_{y}^{A} \leqslant \delta_{y} \leqslant 6 \delta_{y}^{A}$.

By Proposition 3.3 and Lemmas 3.1 and 3.4, we get

$$
\begin{aligned}
& \int_{B} M(|x-z|) P_{A}(y, z) \mathrm{d} z \leqslant C_{4} \int_{B} M(|x-z|) \frac{V\left(\delta_{y}^{A}\right)}{V\left(\delta_{z}^{A}\right)|y-z|} \mathrm{d} z \\
& \quad \leqslant c_{1} \frac{V\left(\delta_{y}\right)}{|x-y|} \int_{B} \frac{M(|x-z|)}{V\left(\delta_{z}^{A}\right)} \mathrm{d} z \leqslant c_{2} \frac{V\left(\delta_{y}\right) V\left(\delta_{x}^{A}\right)}{|x-y| \delta_{x}^{A}} \leqslant c_{3} \frac{V\left(\delta_{y}\right) V\left(\delta_{x}\right)}{|x-y| \delta_{x}} \leqslant c_{4} \frac{G(x, y)}{\delta_{x}} .
\end{aligned}
$$

Since constants $c_{1}, \ldots, c_{4}$ depend on $D$ only via constants $C_{2}, C_{4}$ and $C_{6}$, the proof is completed.

Theorem 3.10 There is a constant $C_{10}=C_{10}\left(\underline{\sigma}, \operatorname{diam}(D) / r_{0}, 1 \vee \operatorname{diam}(D)\right)$ such that

$$
\left|\partial_{x} G(x, y)\right| \leqslant C_{10} \frac{G(x, y) \wedge K(|x-y|)}{|x-y| \wedge \delta_{x}}, \quad x, y \in D
$$

Proof First, assume $|x-y|>\delta_{x} / 2$. By Corollary 3.6, Lemma 3.9 and monotonicity of $K$, we have

$$
\left|\partial_{x} G(x, y)\right| \leqslant c_{1}\left(\frac{G(x, y)}{\delta_{x}} \wedge \frac{K\left(|x-y| \wedge \delta_{x}\right)}{|x-y| \wedge \delta_{x}}\right) \leqslant c_{1} \frac{G(x, y) \wedge K(|x-y|)}{|x-y| \wedge \delta_{x}} .
$$

Now, we show the existence of a constant $c$ such that

$$
\frac{G(x, y)}{|x-y|} \geqslant c M(|x-y|),
$$


when $|x-y| \leqslant \delta_{x} / 2$. In this case $\delta_{x} \approx \delta_{y}$ and therefore, by Lemma 3.1,

$$
G(x, y) \stackrel{C_{2}}{\approx} \frac{V^{2}\left(\delta_{x}\right)}{\delta_{x}}
$$

Since $\underline{\alpha}_{1}>1$, by (2.7), we obtain that $s \mapsto V^{2}(s) / s$ is almost increasing (bounded from below by an increasing function). Hence, we get the claim.

We end this section with the proof of the uniform integrability of $\partial_{z} G(z, y)$.

Lemma 3.11 The function $\partial_{z} G(z, y)$ is uniformly in $y$ integrable against $|b(z)| \mathrm{d} z$.

Proof It is enough to show that

$$
\lim _{N \rightarrow \infty} \sup _{y \in \mathbb{R}} \int_{\left|\partial_{z} G(z, y)\right|>N}\left|\partial_{z} G(z, y)\right||b(z)| \mathrm{d} z=0 .
$$

Let $N>0$ and $r_{N}=\inf \left\{r>0: M(r) \leqslant N / C_{8}\right\} \wedge r_{0}$. Note that $\lim _{r \rightarrow 0} M(r)=\infty$; hence, $r_{N} \rightarrow 0$ as $N \rightarrow \infty$. Fix $y \in \mathbb{R}$, and take $N$ such that $r_{N} \leqslant r_{0}$. By (3.14), $\left\{z:\left|\partial_{z} G(z, y)\right|>N\right\} \subset\left\{z: M\left(\delta_{z}\right)>N / C_{8}\right\} \cup\left\{z: M(|z-y|)>N / C_{8}\right\} \subset\{z:$ $\left.\delta_{z}<r_{N}\right\} \cup\left\{z:|y-z|<r_{N}\right\}$. Let

$$
K_{r}=\sup _{y \in \mathbb{R}} \int_{B(y, r)} M(|y-z|)|b(z)| \mathrm{d} z .
$$

By Corollary 3.6 and monotonicity of $M(\cdot)$, we have

$$
\begin{aligned}
& \int_{\left|\partial_{z} G(z, y)\right|>N}\left|\partial_{z} G(z, y)\right||b(z)| \mathrm{d} z \\
& \leqslant C_{8}\left(\int_{\delta_{z}<r_{N}} M\left(\delta_{z}\right)|b(z)| \mathrm{d} z+\int_{|z-y|<r_{N}} M(|z-y|)|b(z)| \mathrm{d} z\right) \\
& \leqslant C_{8}\left(\sum_{k=1}^{2 n} \int_{B\left(a_{k}, r_{N}\right)} M\left(\left|a_{k}-z\right|\right)|b(z)| \mathrm{d} z+\int_{|z-y|<r_{N}} M(|z-y|)|b(z)| \mathrm{d} z\right) \\
& \leqslant(2 n+1) C_{8} K_{r_{N}},
\end{aligned}
$$

where $D=\bigcup_{k=1}^{n}\left(a_{2 k-1}, a_{2 k}\right)$. By (2.15), $\lim _{N \rightarrow \infty} K_{r_{N}}=0$, which completes the proof. 


\subsection{G Inequalities}

Now, we apply the estimates of the Green function and its derivative to obtain the following $3 G$-type inequalities.

Proposition 3.12 There is a constant $C_{11}=C_{11}(\underline{\sigma}, 1 \vee \operatorname{diam}(\mathrm{D}))$ such that

$$
\frac{G(x, z) G(z, y)}{G(x, y)} \leqslant C_{11} V\left(\delta_{z}\right)\left(\frac{G(x, z)}{V\left(\delta_{x}\right)} \vee \frac{G(z, y)}{V\left(\delta_{y}\right)}\right) .
$$

Proof For $x, y \in D$, we define

$$
\mathcal{G}(x, y)=\frac{G(x, y)}{V\left(\delta_{x}\right) V\left(\delta_{y}\right)} .
$$

It suffices to prove that for any $x, y, z \in D$, we have

$$
\mathcal{G}(x, z) \wedge \mathcal{G}(z, y) \leqslant c \mathcal{G}(x, y) \text {. }
$$

By Lemma 3.1,

$$
\begin{aligned}
C_{3}^{-1}(\mathcal{G}(x, z) \wedge \mathcal{G}(z, y)) & \leqslant \frac{1}{\left(\delta_{x} \delta_{z}\right)^{1 / 2}} \wedge \frac{1}{|x-z|} \wedge \frac{1}{\left(\delta_{z} \delta_{y}\right)^{1 / 2}} \wedge \frac{1}{|z-y|} \\
& =\frac{1}{\left(\delta_{x} \delta_{y}\right)^{1 / 2}}\left(\frac{\delta_{x} \wedge \delta_{y}}{\delta_{z}}\right)^{1 / 2} \wedge \frac{1}{|x-z| \vee|z-y|}
\end{aligned}
$$

If $\frac{\delta_{x} \wedge \delta_{y}}{\delta_{z}} \leqslant 2$, Lemma 3.1 implies

$$
\mathcal{G}(x, z) \wedge \mathcal{G}(z, y) \leqslant 2 C_{3}\left(\frac{1}{\left(\delta_{x} \delta_{y}\right)^{1 / 2}} \wedge \frac{1}{|x-y|}\right) \leqslant 2 C_{3}^{2} \mathcal{G}(x, y) .
$$

If $\frac{\delta_{x} \wedge \delta_{y}}{\delta_{z}} \geqslant 2$, then $\sqrt{\delta_{x} \delta_{y}} \leqslant \delta_{x} \vee \delta_{y} \leqslant 2(|x-z| \vee|y-z|)$ and in consequence

$$
\mathcal{G}(x, z) \wedge \mathcal{G}(z, y) \leqslant \frac{C_{3}}{|x-z| \vee|z-y|} \leqslant 2 C_{3}^{2} \mathcal{G}(x, y) .
$$

Lemma 3.13 There is a constant $C_{12}=C_{12}\left(\underline{\sigma}\right.$, diam(D) $\left./ r_{0}, 1 \vee \operatorname{diam}(\mathrm{D})\right)$ such that for any $x, y, z \in D$, we have

$$
\frac{G(x, z)\left|\partial_{z} G(z, y)\right|}{G(x, y)} \leqslant C_{12} M\left(\delta_{z} \wedge|y-z|\right) .
$$


Proof Note that $\delta_{z}^{2} \leqslant 4\left(\delta_{x} \delta_{z} \vee|x-z|^{2}\right)$. Indeed, for $\delta_{z} \leqslant 2 \delta_{x}, \delta_{z}^{2} \leqslant 2 \delta_{z} \delta_{x}$. Next, for $\delta_{z}>2 \delta_{x}, \delta_{x} \leqslant|x-z|$ and consequently, by triangle inequality, $\delta_{z}^{2} \leqslant\left(\delta_{x}+|x-z|\right)^{2} \leqslant$ $4|x-z|^{2}$. Hence,

$$
\frac{V\left(\delta_{z}\right) G(x, z)}{V\left(\delta_{x}\right)} \approx V\left(\delta_{z}\right)^{2}\left(\frac{1}{\left(\delta_{x} \delta_{z}\right)^{1 / 2}} \wedge \frac{1}{|x-z|}\right) \leqslant 2 \frac{V\left(\delta_{z}\right)^{2}}{\delta_{z}}
$$

By Proposition 3.12 and (3.18),

$$
\frac{G(x, z) G(z, y)}{G(x, y)} \leqslant C_{11}\left(\frac{V\left(\delta_{z}\right) G(x, z)}{V\left(\delta_{x}\right)}\right) \vee\left(\frac{V\left(\delta_{z}\right) G(z, y)}{V\left(\delta_{y}\right)}\right) \leqslant c_{1} \frac{V\left(\delta_{z}\right)^{2}}{\delta_{z}},
$$

where $c_{1}=2 C_{3} C_{11}$. For $\delta_{z}<2|y-z|$, by Lemma 3.9 and (3.19), we get

$$
\frac{G(x, z)\left|\partial_{z} G(z, y)\right|}{G(x, y)} \leqslant C_{9} \frac{G(x, z)}{G(x, y)} \frac{G(z, y)}{\delta_{z}} \leqslant c_{2} M\left(\delta_{z}\right)
$$

where $c_{2}=c_{1} C_{9}$. Now, let $\delta_{z} \geqslant 2|z-y|$. Note that $\delta_{z} \approx \delta_{y}$ and in consequence $G(z, y) \approx V^{2}\left(\delta_{z}\right) / \delta_{z}$. Hence, by (3.14) and (3.19), we have

$$
\begin{aligned}
\frac{G(x, z)\left|\partial_{z} G(z, y)\right|}{G(x, y)} & \leqslant C_{8} \frac{G(x, z)}{G(x, y)} M(|z-y|) \leqslant \frac{c_{3} V^{2}\left(\delta_{z}\right)}{G(z, y) \delta_{z}} M(|z-y|) \\
& \leqslant c_{4} M(|z-y|)
\end{aligned}
$$

where $c_{3}=c_{1} C_{8}$ and $c_{4}=c_{3}\left(\frac{2 C_{1}}{\underline{c}}\right) \sqrt{\frac{3}{2}}$. Now, by (2.5), the assertion of the lemma holds.

For $x, y \in D$, we define

$$
\kappa(x, y)=\int_{D}\left|b(z) \frac{G(x, z) \partial_{z} G(z, y)}{G(x, y)}\right| \mathrm{d} z
$$

Lemma 3.14 Let $\lambda<\infty, R<1$. There is a constant $C_{13}=C_{13}(\underline{\sigma}, b, \lambda, R)$ such that if $\operatorname{diam}(D) / r_{0}(D) \leqslant \lambda$ and $\operatorname{diam}(D) \leqslant R$, then

$$
\kappa(x, y) \leqslant C_{13}, \quad x, y \in D .
$$

Furthermore, $C_{13} \rightarrow 0$ as $R \rightarrow 0$.

Proof Since $b \in \mathcal{K}_{1}$, (3.21) follows by Lemma 3.13 and (2.15). 


\section{Green Function of $\tilde{\mathcal{L}}$}

Following [8] and [27] we recursively define, for $t>0$ and $x, y \in \mathbb{R}$,

$$
\begin{aligned}
& p_{0}(t, x, y)=p(t, x, y), \\
& p_{n}(t, x, y)=\int_{0}^{t} \int_{\mathbb{R}} p_{n-1}(t-s, x, z) b(z) \partial_{z} p(s, z, y) \mathrm{d} z \mathrm{~d} s, \quad n \geqslant 1,
\end{aligned}
$$

and we let

$$
\tilde{p}(t, x, y)=\sum_{n=0}^{\infty} p_{n}(t, x, y) .
$$

By [27, Theorem 1.1] the series converges absolutely, $\tilde{p}$ is a continuous probability transition density function, and

$$
c_{T}^{-1} p(t, x, y) \leqslant \tilde{p}(t, x, y) \leqslant c_{T} p(t, x, y), \quad x, y \in \mathbb{R}, 0<t<T,
$$

where $c_{T} \rightarrow 1$ if $T \rightarrow 0$, see [8, Theorem 2].

By Chapman-Kolmogorov equation (see [27]), there is $C_{14}>0$ such that

$$
C_{14}^{-1-t} p(t, x-y) \leqslant \tilde{p}(t, x, y) \leqslant C_{14}^{t+1} p(t, x-y), \quad t>0, x, y \in \mathbb{R} .
$$

We let $\tilde{\mathbb{P}}, \tilde{\mathbb{E}}$ be the Markov distributions and expectations defined by the transition density $\tilde{p}$ on the canonical path space. By Hunt formula,

$$
\tilde{p}_{D}(t, x, y)=\tilde{p}(t, x, y)-\tilde{\mathbb{E}}^{x}\left[\tau_{D}<t ; \tilde{p}\left(t-\tau_{D}, X_{\tau_{D}}, y\right)\right]
$$

Except for the symmetry, $\tilde{p}_{D}$ has analogous properties as $p_{D}$, i.e., the ChapmanKolmogorov equation holds

$$
\int_{\mathbb{R}^{d}} \tilde{p}_{D}(s, x, z) \tilde{p}_{D}(t, z, y) \mathrm{d} z=\tilde{p}_{D}(s+t, x, y), \quad s, t>0, x, y \in \mathbb{R}
$$

$0 \leqslant \tilde{p}_{D}(t, x, y) \leqslant \tilde{p}(t, x, y)$ and $\tilde{p}_{D}$ is jointly continuous on $(0, \infty) \times D \times D$ (see [19, Lemma 2.3]).

We denote by $\tilde{G}_{D}(x, y)$ the Green function of $\tilde{\mathcal{L}}=\mathcal{L}+b \partial$ on $D$,

$$
\tilde{G}_{D}(x, y)=\int_{0}^{\infty} \tilde{p}_{D}(t, x, y) \mathrm{d} t
$$

As for $G$, from now on, every time we will mention the Green function $\tilde{G}$, it should be understood as the Green function of $\tilde{\mathcal{L}}$ on $D$, and then $\tilde{G}=\tilde{G}_{D}$.

For $x \in D^{c}$ or $y \in D^{c}$, we observe both $\tilde{p}_{D}(t, x, y)=0$ and $\tilde{G}(x, y)=0$. Indeed, since $D$ is a $C^{1,1}$ set, for any $x \in D^{c}$ (or $y \in D^{c}$ ), we can find some interval 
$B_{x}=\overline{B\left(x_{0}, r_{0} / 2\right)} \subset D^{c}$ such that $x \in B_{x}$. Since $p(t, \cdot)$ is unimodal, by (4.3), we have

$$
0<c<\mathbb{P}^{x}\left(X_{t} \in B_{x}\right) \approx \tilde{\mathbb{P}}^{x}\left(X_{t} \in B_{x}\right) \leqslant \tilde{\mathbb{P}}^{x}\left(\tau_{D}<t\right)
$$

for every $t<1$. This implies $c<\tilde{\mathbb{P}}^{x}\left(\tau_{D}=0\right)$ and by Blumenthal's zero-one law ([14]), we have $\tilde{\mathbb{P}}^{x}\left(\tau_{D}=0\right)=1$. Thus, by (4.4), we get the assertion.

By (4.2), we have

$$
\lim _{t \rightarrow 0} \frac{\tilde{p}(t, x, y)}{t}=\lim _{t \rightarrow 0} \frac{p(t, x, y)}{t}=v(y-x) .
$$

Thus, the intensity of jumps of the canonical process $X_{t}$ under $\tilde{\mathbb{P}}^{x}$ is the same as under $\mathbb{P}^{x}$. Accordingly, we obtain the following description.

Lemma 4.1 The $\tilde{\mathbb{P}}^{x}$-distribution of $\left(\tau_{D}, X_{\tau_{D}}\right)$ on $(0, \infty) \times(\bar{D})^{c}$ has density

$$
\int_{D} \tilde{p}_{D}(u, x, y) v(z-y) \mathrm{d} y, \quad u>0, \delta_{z}>0 .
$$

We define the Poisson kernel of $D$ for $\tilde{\mathcal{L}}$,

$$
\tilde{P}_{D}(x, y)=\int_{D} \tilde{G}(x, z) v(|y-z|) \mathrm{d} z, \quad x \in D, y \in D^{c} .
$$

By (4.5), (4.7) and (4.6), we have

$$
\tilde{\mathbb{P}}^{x}\left(X_{\tau_{D}} \in A\right)=\int_{A} \int_{D} \tilde{G}(x, z) v(|y-z|) \mathrm{d} z \mathrm{~d} y=\int_{A} \tilde{P}_{D}(x, y) \mathrm{d} y,
$$

if $A \subset(\bar{D})^{c}$. For the case of $A \subset \partial D$, we refer the reader to (5.8) below.

Lemma $4.2 \tilde{G}(x, y)$ is continuous and

$$
\tilde{G}(x, y) \leqslant C_{15}, \quad x, y \in \mathbb{R},
$$

where $C_{15}=C_{15}(\sigma, b, \operatorname{diam}(D))$.

Proof In the same way as in [9, Lemma 7], we get that there are positive constants $c$ and $C$ such that

$$
\tilde{p}_{D}(t, x, y) \leqslant C e^{-c t}, \quad t>1, \quad x, y \in \mathbb{R} .
$$


By (4.5), (4.2) and (4.9), we obtain

$$
\begin{aligned}
\tilde{G}(x, y) & \leqslant \int_{0}^{1} C_{14} p(t, x, y) \mathrm{d} t+\int_{1}^{\infty} C e^{-c t} \mathrm{~d} t \\
& \leqslant \int_{0}^{1} p(t, 0,0) \mathrm{d} t+\int_{1}^{\infty} C e^{-c t} \mathrm{~d} t \\
& \leqslant c_{1}+C / c
\end{aligned}
$$

where $c_{1}$ is finite bound for $\int_{0}^{1} p(t, 0,0) \mathrm{d} t$. We put $C_{15}=c_{1}+C / c$. By (4.5), continuity of $\tilde{p}_{D}$ and the dominated convergence theorem, $\tilde{G}(x, y)$ is continuous.

By Lemmas 3.11 and 4.2, for every $x \in D$, the function

$$
f_{x}(y):=\tilde{G}(x, y)-G(x, y)-\int_{D} \tilde{G}(x, z) b(z) \partial_{z} G(z, y) \mathrm{d} z
$$

is well defined, integrable and bounded on $\mathbb{R}$. Hence, following [19, Theorem 3.1], we obtain the following perturbation formula (for the proof see [19]).

Lemma 4.3 Let $x, y \in \mathbb{R}$. We have

$$
\tilde{G}(x, y)=G(x, y)+\int_{D} \tilde{G}(x, z) b(z) \partial_{z} G(z, y) \mathrm{d} z
$$

\section{Proof of Theorem 1.1}

First, we will prove the comparability of $G$ and $\tilde{G}$ for small sets $D$ from the $C^{1,1}$ class. For this purpose, we could consider the perturbed series for $\tilde{G}$ as presented in [19]. We could define by induction the functions $G_{n}$ and show the convergence and estimates of the series

$$
\tilde{G}(x, y)=\sum_{n=0}^{\infty} G_{n}(x, y) .
$$

However, since $\tilde{G}$ is bounded, we present a simpler proof of the following lemma (compare [19, Lemma 3.11]).

Lemma 5.1 Let $b \in \mathcal{K}_{1}$ and $\lambda>0$. There is $\varepsilon=\varepsilon(\underline{\sigma}, b, \lambda)>0$ such that if $\operatorname{diam}(D) / r_{0}(D) \leqslant \lambda$ and $\operatorname{diam}(D) \leqslant \varepsilon$, then

$$
\frac{1}{2} G(x, y) \leqslant \tilde{G}(x, y) \leqslant \frac{3}{2} G(x, y), \quad x, y \in \mathbb{R} .
$$


Proof By Lemma 3.14, there exists $\varepsilon_{1}>0$ such that if $\operatorname{diam}(D)<\varepsilon_{1}$, then

$$
\int_{D} G(x, z)\left|\partial_{z} G(z, y) b(z)\right| \mathrm{d} z \leqslant C_{13} G(x, y),
$$

and $C_{13}<\frac{1}{3}$. Let $0<\eta<1$. By Lemma 3.11, there exists $\varepsilon_{2}>0$ such that if $\operatorname{diam}(D)<\varepsilon_{2}$, then

$$
\sup _{y \in \mathbb{R}} \int_{D}\left|\partial_{z} G(z, y) b(z)\right| \mathrm{d} z \leqslant \eta
$$

We put $\varepsilon=\min \left(\varepsilon_{1}, \varepsilon_{2}\right)$ and $\operatorname{diam}(D) \leqslant \varepsilon$. By Lemma 4.3,

$$
\begin{aligned}
\tilde{G}(x, y) & \leqslant G(x, y)+\int_{D} \tilde{G}(x, z)\left|b(z) \partial_{z} G(z, y)\right| \mathrm{d} z \\
& \leqslant G(x, y)+C_{15} \eta .
\end{aligned}
$$

By putting the estimates of $\tilde{G}$ from (5.4) into (5.3) and applying (5.2), we get

$$
\begin{aligned}
\tilde{G}(x, y) & \leqslant G(x, y)+\int_{D}\left(G(x, y)+C_{15} \eta\right)\left|b(z) \partial_{z} G(z, y)\right| \mathrm{d} z \\
& \leqslant G(x, y)\left(1+C_{13}\right)+C_{15} \eta^{2} .
\end{aligned}
$$

By induction,

$$
\tilde{G}(x, y) \leqslant G(x, y)\left(1+C_{13}+\cdots+C_{13}^{n-1}\right)+C_{15} \eta^{n} .
$$

Now, taking $n \rightarrow \infty$, for every $x, y \in D$, we obtain

$$
\tilde{G}(x, y) \leqslant G(x, y) \frac{1}{1-C_{13}} .
$$

Since $C_{13}<\frac{1}{3}$, by Lemma 4.3, (5.6) and (5.2), we get

$$
\begin{aligned}
\tilde{G}(x, y) & \geqslant G(x, y)-\frac{1}{1-C_{13}} \int_{D} G(x, z)\left|b(z) \partial_{z} G(z, y)\right| \mathrm{d} z \\
& \geqslant G(x, y)\left(1-\frac{C_{13}}{1-C_{13}}\right) .
\end{aligned}
$$

We note that the comparison constants in the proof above will improve to 1 if $\operatorname{diam}(D) \rightarrow 0$ and the distortion of $D$ is bounded. By (4.8),

$$
\tilde{\mathbb{P}}^{x}\left(X_{\tau_{D}} \in A\right) \stackrel{C_{16}}{\approx} \mathbb{P}^{x}\left(X_{\tau_{D}} \in A\right), \quad x \in D, \quad A \subset(\bar{D})^{c},
$$

where $C_{16}=C_{16}(\underline{\sigma}, b, \lambda, \operatorname{diam}(D))$ and $\operatorname{diam}(D)<\varepsilon$ from Lemma 5.1. 
Following [9, Proof of Lemma 14], we obtain that the boundary of our general $C^{1,1}$ open set $D$ is not hit at the first exit, i.e.,

$$
\tilde{\mathbb{P}}^{x}\left(X_{\tau_{D}} \in \partial D\right)=0, \quad x \in D
$$

Hence, in the context of Lemma 5.1, the $\tilde{\mathbb{P}}^{x}$ distribution of $X_{\tau_{D}}$ is absolutely continuous with respect to the Lebesgue measure and has the density function

$$
\tilde{P}_{D}(x, y) \approx P_{D}(x, y), \quad y \in D^{c},
$$

provided $x \in D$. This follows from (4.8) and (5.8).

The definition of $\tilde{\mathcal{L}}$-harmonicity is analogous to that of $\mathcal{L}$-harmonicity.

Definition 5 We say that a function $f: \mathbb{R} \rightarrow \mathbb{R}$ is $\tilde{\mathcal{L}}$-harmonic on an open bounded set $D \subset \mathbb{R}$, if for any open $F \subset \bar{F} \subset D$ and $x \in F$

$$
f(x)=\tilde{\mathbb{E}}^{x} f\left(X_{\tau_{F}}\right) .
$$

We say that a function $f$ is regular $\tilde{\mathcal{L}}$-harmonic on an open bounded set $D \subset \mathbb{R}$, if for every $x \in D$

$$
f(x)=\tilde{\mathbb{E}}^{x} f\left(X_{\tau_{D}}\right) .
$$

Following [9] and [19], we get the following Harnack inequality.

Lemma 5.2 (Harnack inequality for $\tilde{\mathcal{L}}$ ). Let $x, y \in \mathbb{R}, 0<s<1$ and $k \in \mathbb{N}$ satisfy $|x-y| \leqslant 2^{k}$ s. Let $u$ be nonnegative in $\mathbb{R}$ and $\tilde{\mathcal{L}}$-harmonic in $B(x, s) \cup B(y, s)$. There is $C_{17}=C_{17}(\bar{\alpha}, \bar{C}, b)$ such that

$$
C_{17}^{-1} 2^{-k(1+\bar{\alpha})} u(x) \leqslant u(y) \leqslant C_{17} 2^{k(1+\bar{\alpha})} u(x) .
$$

We obtain a boundary Harnack principle for $\mathcal{L}$ and general $C^{1,1}$ sets $D$. See the proof of [19, Lemma 4.3].

Lemma 5.3 (BHP). Let $z \in \partial D, 0<r \leqslant r_{0}(D)$, and $0<q<1$. If $\tilde{u}, \tilde{v}$ are nonnegative in $\mathbb{R}$, regular $\tilde{\mathcal{L}}$-harmonic in $D \cap B(z, r)$, vanish on $D^{c} \cap B(z, r)$ and satisfy $\tilde{u}\left(x_{0}\right)=\tilde{v}\left(x_{0}\right)$ for some $x_{0} \in D \cap B(z, q r)$ then

$$
C_{18}^{-1} \tilde{v}(x) \leqslant \tilde{u}(x) \leqslant C_{18} \tilde{v}(x), \quad x \in D \cap B(z, q r),
$$

with $C_{18}=C_{18}\left(\sigma, b, q, r_{0}(D)\right)$.

Now, we have all the tools necessary to prove the main result of our paper. Since in the proof we follow the idea from [9], we only give its basic steps (for details see [9, Proof of Theorem 1]). 
Proof of Theorem 1.1 By (4.10), we have the estimate

$$
\tilde{G}(x, y) \leqslant G(x, y)+\int_{D}\left|\tilde{G}(x, z) \partial_{z} G(z, y)\right||b(z)| \mathrm{d} z, \quad x, y \in D .
$$

We consider $\eta<1$, say $\eta=1 / 2$. By Lemmas 3.11 and 3.14 there is a constant $r>0$ so small that

$$
\int_{D^{r}}\left|\partial_{z} G(z, y) b(z)\right| \mathrm{d} z<\eta, \quad y \in D
$$

and

$$
\int_{D^{r}} \frac{G(x, z)\left|\partial_{z} G(z, y)\right|}{G(x, y)}|b(z)| \mathrm{d} z<\eta, \quad y \in D,
$$

where $D^{r}=\left\{z \in D: \delta_{z} \leqslant r\right\}$. We denote

$$
\rho=\left[\varepsilon \wedge r_{0}(D) \wedge r\right] / 16
$$

with $\varepsilon=\varepsilon(\psi, b, \lambda, \operatorname{diam}(D))$ of Lemma 5.1.

To prove (1.2) we consider $x$ and $y$ in a partition of $D \times D$.

First, we consider $y$ far from the boundary of $D$, say $\delta_{y} \geqslant \rho / 4$.

- For $|x-y| \leqslant \rho / 8$, we consider the interval $B=B(y, \rho / 4)$. Thus, by Lemmas 3.1, 4.2 and 5.1, we have $G(x, y) \approx G_{B}(x, y) \approx \tilde{G}_{B}(x, y) \approx \tilde{G}(x, y)$.

- If $\rho / 8<\delta_{x}$, we use Harnack inequalities for $\mathcal{L}$ and $\tilde{\mathcal{L}}$.

- For $\delta_{x}<\rho / 8$, we use boundary Harnack principles (see Lemma 5.3, [28, Theorem 2.18]).

Next, suppose that $\delta_{y} \leqslant \rho / 4$. Here, the difficulty lies in the fact $\tilde{G}$ is non-symmetric. In the proof of lower bounds, we consider two cases: $x$ close to $y$ and $x$ far away from $y$.

- In the case $|x-y| \leqslant \rho$, we consider an interval $F$ such that $\delta_{x}=\delta_{x}^{F}$ and $\delta_{y}=\delta_{y}^{F}$. Then $\tilde{G}(x, y) \geqslant \tilde{G}_{F}(x, y) \approx G_{F}(x, y) \approx G(x, y)$ (see Lemmas 3.1 and 5.1).

- For $|x-y|>\rho$ and $\delta_{x} \geqslant \rho / 4$ we use Harnack inequalities. For $\delta_{x} \leqslant \rho / 4$, we use boundary Harnack principles.

In the next step, we prove the upper bound in (1.2) for $\delta_{x} \geqslant \rho / 4$. We have already proved that for $y \in D \backslash D^{r}$,

$$
c_{1}^{-1} G(x, y) \leqslant \tilde{G}(x, y) \leqslant c_{1} G(x, y) .
$$

By (3.20), Lemmas 3.14, 4.2, (5.12) and (5.13),

$$
\begin{aligned}
\tilde{G}(x, y) & \leqslant A G(x, y)+\int_{D_{r}} \tilde{G}(x, z)\left|\partial_{z} G(z, y) b(z)\right| \mathrm{d} z, \\
& \leqslant A G(x, y)+B(x),
\end{aligned}
$$

where $A=1+c_{1} C_{3}$ and $B(x)=\eta C_{7}$. Now, plugging (5.16) into (5.15), and using (5.13), (5.14) and induction, we get for $n=0,1, \ldots$,

$$
\tilde{G}(x, y) \leqslant A\left(1+\eta+\cdots+\eta^{n}\right) G(x, y)+\eta^{n} B(x) .
$$


In consequence,

$$
\tilde{G}(x, y) \leqslant \frac{A}{1-\eta} G(x, y) .
$$

Finally, we prove the upper bound in (1.2) when $\delta_{x}<\rho / 4$.

- If $|x-y|>\rho$, we use boundary Harnack principles.

- For $|x-y| \leqslant \rho$, consider the same interval $F$ as above. We have

$$
\tilde{G}(x, y)=\tilde{G}_{F}(x, y)+\int_{D \backslash F} \tilde{P}_{F}(x, z) \tilde{G}(z, y) \mathrm{d} z .
$$

By Lemma 5.1 and (5.9), $\tilde{G}_{F}(x, y) \approx G_{F}(x, y)$ and $\tilde{P}_{F}(x, z) \approx P_{F}(x, z)$. We already know that for $|z-y|>\rho, \tilde{G}(z, y) \approx G(z, y)$. Thus,

$$
\tilde{G}(x, y) \approx G_{F}(x, y)+\int_{D \backslash F} P_{F}(x, z) G(z, y) \mathrm{d} z=G(x, y) .
$$

The proof of Theorem 1.1 is complete.

Open Access This article is distributed under the terms of the Creative Commons Attribution 4.0 International License (http://creativecommons.org/licenses/by/4.0/), which permits unrestricted use, distribution, and reproduction in any medium, provided you give appropriate credit to the original author(s) and the source, provide a link to the Creative Commons license, and indicate if changes were made.

\section{References}

1. Bertoin, J.: Lévy Processes, Volume 121 of Cambridge Tracts in Mathematics. Cambridge University Press, Cambridge (1996)

2. Blumenthal, R.M., Getoor, R.K.: Markov Processes and Potential Theory. Pure and Applied Mathematics, vol. 29. Academic Press, New York (1968)

3. Blumenthal, R.M., Getoor, R.K., Ray, D.B.: On the distribution of first hits for the symmetric stable processes. Trans. Am. Math. Soc. 99, 540-554 (1961)

4. Bogdan, K., Butko, Y., Szczypkowski, K.: Majorization, 4G theorem and Schrödinger perturbations. J. Evol. Equ. 16(2), 241-260 (2016)

5. Bogdan, K., Grzywny, T., Ryznar, M.: Density and tails of unimodal convolution semigroups. J. Funct. Anal. 266(6), 3543-3571 (2014)

6. Bogdan, K., Grzywny, T., Ryznar, M.: Dirichlet heat kernel for unimodal Lévy processes. Stoch. Process. Appl. 124(11), 3612-3650 (2014)

7. Bogdan, K., Hansen, W., Jakubowski, T.: Time-dependent Schrödinger perturbations of transition densities. Stud. Math. 189(3), 235-254 (2008)

8. Bogdan, K., Jakubowski, T.: Estimates of heat kernel of fractional Laplacian perturbed by gradient operators. Commun. Math. Phys. 271(1), 179-198 (2007)

9. Bogdan, K., Jakubowski, T.: Estimates of the Green function for the fractional Laplacian perturbed by gradient. Potential Anal. 36(3), 455-481 (2012)

10. Bogdan, K., Komorowski, T.: Principal eigenvalue of the fractional Laplacian with a large incompressible drift. NoDEA Nonlinear Differ. Equ. Appl. 21(4), 541-566 (2014)

11. Caffarelli, L.A., Vasseur, A.: Drift diffusion equations with fractional diffusion and the quasigeostrophic equation. Ann. Math. (2) 171(3), 1903-1930 (2010) 
12. Chen, Z.-Q., Kim, P., Song, R.: Dirichlet heat kernel estimates for fractional Laplacian with gradient perturbation. Ann. Probab. 40(6), 2483-2538 (2012)

13. Chen, Z.-Q., Kim, P., Song, R.: Dirichlet heat kernel estimates for rotationally symmetric Lévy processes. Proc. Lond. Math. Soc. (3) 109(1), 90-120 (2014)

14. Chung, K.L., Walsh, J.B.: Markov Processes, Brownian Motion, and Time Symmetry, Volume 249 of Grundlehren der Mathematischen Wissenschaften [Fundamental Principles of Mathematical Sciences], 2nd edn. Springer, New York (2005)

15. Chung, K.L., Zhao, Z.X.: From Brownian Motion to Schrödinger's Equation, Volume 312 of Grundlehren der Mathematischen Wissenschaften [Fundamental Principles of Mathematical Sciences]. Springer, Berlin (1995)

16. Cranston, M., Zhao, Z.: Conditional transformation of drift formula and potential theory for $\frac{1}{2} \Delta+$ $b(\cdot) \cdot \nabla$. Commun. Math. Phys. 112(4), 613-625 (1987)

17. Graczyk, P., Jakubowski, T., Luks, T.: Martin representation and relative Fatou theorem for fractional Laplacian with a gradient perturbation. Positivity 17(4), 1043-1070 (2013)

18. Grzywny, T.: On Harnack inequality and Hölder regularity for isotropic unimodal Lévy processes. Potential Anal. 41(1), 1-29 (2014)

19. Grzywny, T., Jakubowski, T., Żurek, G.: Green function for gradient perturbation of unimodal Lévy processes. Probab. Math. Stat. 37(1), 119-143 (2017)

20. Grzywny, T., Ryznar, M.: Potential theory of one-dimensional geometric stable processes. Colloq. Math. 129(1), 7-40 (2012)

21. Grzywny, T., Ryznar, M.: Hitting times of points and intervals for symmetric Lévy processes. Potential Anal. 46(4), 739-777 (2017)

22. Grzywny, T., Szczypkowski, K.: Estimates of heat kernels of non-symmetric Lévy processes. ArXiv e-prints: arXiv: 1710.07793

23. Ikeda, N., Watanabe, S.: On some relations between the harmonic measure and the Lévy measure for a certain class of Markov processes. J. Math. Kyoto Univ. 2, 79-95 (1962)

24. Jakubowski, T.: The estimates of the mean first exit time from a ball for the $\alpha$-stable Ornstein-Uhlenbeck processes. Stoch. Process. Appl. 117(10), 1540-1560 (2007)

25. Jakubowski, T.: On Harnack inequality for $\alpha$-stable Ornstein-Uhlenbeck processes. Math. Z. 258(3), 609-628 (2008)

26. Jakubowski, T.: Fractional Laplacian with singular drift. Stud. Math. 207(3), 257-273 (2011)

27. Jakubowski, T., Szczypkowski, K.: Estimates of gradient perturbation series. J. Math. Anal. Appl. 389(1), 452-460 (2012)

28. Kim, P., Song, R., Vondraček, Z.: Martin boundary for some symmetric Lévy processes. In: Chen, Z.-Q., Jacob, N., Takeda, M., Uemura, T. (eds.) Festschrift Masatoshi Fukushima, pp. 307-342. World Scientific, Singapore (2015)

29. Kulczycki, T., Ryznar, M.: Gradient estimates of harmonic functions and transition densities for Lévy processes. Trans. Am. Math. Soc. 368(1), 281-318 (2016)

30. Maekawa, Y., Miura, H.: Upper bounds for fundamental solutions to non-local diffusion equations with divergence free drift. J. Funct. Anal. 264(10), 2245-2268 (2013)

31. Portenko, N.I.: Some perturbations of drift-type for symmetric stable processes. Random Oper. Stoch. Equ. 2(3), 211-224 (1994)

32. Pruitt, W.E.: The growth of random walks and Lévy processes. Ann. Probab. 9(6), 948-956 (1981)

33. Silvestre, L.: Hölder estimates for advection fractional-diffusion equations. Ann. Sc. Norm. Super. Pisa Cl. Sci. (5) 11(4), 843-855 (2012)

34. Silvestre, L., Vicol, V., Zlatoš, A.: On the loss of continuity for super-critical drift-diffusion equations. Arch. Ration. Mech. Anal. 207(3), 845-877 (2013)

35. Watanabe, T.: The isoperimetric inequality for isotropic unimodal Lévy processes. Z. Wahrsch. Verw. Gebiete 63(4), 487-499 (1983)

36. Yano, K.: Excursions away from a regular point for one-dimensional symmetric Lévy processes without Gaussian part. Potential Anal. 32(4), 305-341 (2010)

Publisher's Note Springer Nature remains neutral with regard to jurisdictional claims in published maps and institutional affiliations. 